\title{
Periodogram-Based Carrier Frequency Offset Estimation for Orthogonal Frequency Division Multiplexing Applications
}

\author{
Jing Lei and Tung-Sang $\mathrm{Ng}$ \\ The University of Hong Kong \\ Department of Electrical and Electronics Engineering \\ Pokfulam Road, Hong Kong
}

\begin{abstract}
In this paper, a novel carrier frequency offset (CFO) estimation algorithm is proposed for OFDM applications. The maximum likelihood estimator (MLE) for the CFO is derived, which reveals the relationship between the CFO and the periodogram of the received signal. Theoretical analysis shows that the proposed MLE is statistically efficient. To realize this MLE in practice, a sub-optimal estimator is also introduced in which zero-padded FFT is invoked for implementation. For the objectives of reducing the implementation complexity and broadening the estimation range, a preamble structure comprising nonuniformly-spaced pilot tones is presented. Based on this preamble, the CFO estimation is split into two phases: the coarse estimation is obtained through the correlation between the received spectrum and the original pattern of the preamble; whereas the fine estimation is obtained by comparing the relative magnitude attenuation in the vicinities of those CFO-shifted pilot tones. Both analytical investigations and computer simulations indicate that the accuracy of this simplified sub-optimal estimator is proportional to the oversize ratio of zero-padded FFT, and its estimation range is equal to the bandwidth of OFDM signal. When the oversize ratio is sufficiently high, the performance of the proposed sub-optimal estimator approaches that of the proposed MLE.
\end{abstract}

\section{INTRODUCTION}

Orthogonal frequency division multiplexing (OFDM) is an efficient modulation scheme for the transmission of high-bitrate data. However, OFDM is vulnerable to the carrier frequency offset (CFO) arising from transeceiver oscillator mismatches and/or Doppler shifts [1]. Depending on the application, the CFO may amount to many tens of the subcarrier spacing. However, even the CFO of a fraction of the subcarrier spacing can cause serious performance degradation. Frequency synchronization is therefore one of the most important tasks performed by an OFDM receiver. Generally, the estimation of CFO is split into a coarse phase and a fine phase, and different algorithms are used in these two phases to accommodate their different requirements [2-7].

As for the coarse estimation of CFO, algorithms presented in [2-4] involve the use of a differentially-encoded PN sequence whose overhead is two OFDM symbols. Besides, the CFO must be partially corrected beforehand in [2] and [3] as they can only deal with CFO being an integral multiple of the subcarrier spacing. The techniques in [5-6] are based on an OFDM preamble with periodic time-domain structure, and their estimation range is proportional to the number of periods. Although the estimation range can be broadened by increasing the number of periods, this is achieved at the cost of decreased estimation accuracy. Regarding the fine estimation of CFO, the majority methods are based on the CFO-induced phase rotation. As angle evaluation is unavoidable for these methods, their implementation relies on the use of a lookup table and trade off must be made between the desired precision and the table size.

In this paper, we propose a new CFO estimation algorithm, which employs only one OFDM symbol as the preamble but achieves large estimation range as well as high estimation accuracy. Starting from the likelihood function of the received signal, the close-form expression for the maximum likelihood (ML) estimate of CFO is derived, which reveals the relationship between the $\mathrm{CFO}$ and the periodogram of the received signal. Then, the practical realization of this $\mathrm{ML}$ estimator (MLE) is discussed and a sub-optimal estimator is presented, which can be implemented by invoking zeropadded FFT. In an attempt to reduce the computation burden, a preamble structure similar to [7] is introduced, and binary PN code is utilized in the configuration of the preamble.

The rest of this paper is organized as follows. In the next section, the problem of CFO estimation is formulated in an OFDM receiver and the MLE is derived. The practical realization of this MLE is discussed in section III. Theoretical performance analysis is presented in section IV. Computer simulation results are given in section V. Section VI concludes this paper.

\section{PROBLEM FORMULATION}

We start with the system model of the OFDM signal, which is shown in Fig.1. Let $P=\left[\begin{array}{llll}p_{0} & p_{1} & \ldots & p_{M-1}\end{array}\right]^{T}$ denote the data block to be transmitted, where (. $)^{T}$ is the transposition operator. OFDM modulation is implemented by invoking $N$ point IFFT. Usually, due to the need to avoid adjacent channel interference (ACI), virtual carriers are used. Without loss of generality, we assume subchannels numbered from 0 to $M-1$ are employed for data transmission and define $W_{M}=\left[\begin{array}{llll}w_{0} & w_{1} & \ldots & w_{M-1}\end{array}\right]$ as the submatrix of the IFFT matrix $W$, where $w_{i}$ is the $i$ th column of $W$. Using matrix representation, the vector of time-domain samples is given by

$$
S=W_{M} P \text {. }
$$

To cope with the inter-symbol-interference induced by multipath fading, a cyclic prefix (CP), whose length is longer than the multipath delay spread, is inserted before $S$. Then, the baseband samples are pulse-shaped, up-converted to the 


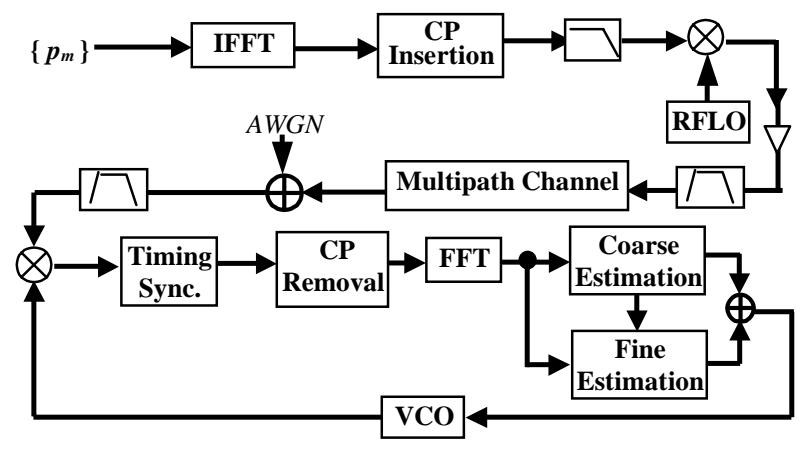

Fig.1 System model of the proposed CFO estimation method for OFDM applications

radio frequency $(\mathrm{RF})$ and transmitted over the multipath channel. Additive white Gaussian noise (AWGN) is also introduced in this channel. At the receiver, the signal is down converted by a local VCO and $f_{d}$ is assumed to be the actual CFO normalized by the subcarrier spacing $\Delta f$. Symbol timing synchronization (represented by "Timing Sync." block in Fig.1) is performed next and the CP is removed. Finally, the received samples can be given by

$$
\begin{aligned}
R & =\left[\begin{array}{llll}
r_{0} & r_{1} & \cdots & r_{N-1}
\end{array}\right]^{T} \\
& =\Omega\left(f_{d}\right) W_{M} H_{f} P+\xi,
\end{aligned}
$$

where $\Omega\left(f_{d}\right)=\operatorname{diag}\left\{1, \exp \left(j 2 \pi f_{d} / N\right) \ldots \exp \left[j 2 \pi(N-1) f_{d} / N\right]\right\}$ is a diagonal matrix, $H_{f}=\operatorname{diag}\left\{h_{0} h_{1} \ldots h_{M-l}\right\}$ is a complex matrix with $h_{i}$ reflecting the channel frequency response at the $i$ th subcarrier, and $\xi=\left[\begin{array}{llll}n_{0} & n_{1} & \ldots & n_{N-1}\end{array}\right]^{T}$ is a vector of complex white Gaussian noise with zero mean and covariance matrix

$$
C_{\xi}=E\left\{\xi \xi^{H}\right\}=\sigma^{2} I_{N},
$$

where $(.)^{H}$ denotes Hermitian transposition and $I_{N}$ is an $N \times N$ identity matrix.

Matrices $\Omega\left(f_{d}\right)$ and $H_{f}$ are both unknown but deterministic. For the convenience of discussion, let $\Xi=\Omega\left(f_{d}\right) W_{M}$ and $\Gamma=H_{f} P$. Given $P, \Gamma$ is a linear function of $H_{f}$; while $\Xi$ is a nonlinear function of $f_{d}$, which can be rewritten as $\Xi=\left[\begin{array}{llll}e_{0} & e_{1} & \ldots & e_{M-1}\end{array}\right]$, with $e_{i}=\left\{1 \exp \left[j 2 \pi\left(i-1+f_{d}\right) / N\right] \quad \ldots \quad \exp \left[j 2 \pi\left(i-1+f_{d}\right)(N-1) / N\right]\right\}$. For a fixed pair of $\left(f_{d}, \Gamma\right)$, the probability density function of $R$ conditioned on $\Xi$ and $\Gamma$ is given by

$$
f\{R \mid \Xi, \Gamma\}=\left(\pi \sigma^{2}\right)^{-N} \exp \left\{\|R-\Xi \Gamma\|^{2} \sigma^{-2}\right\},
$$

where \|\|$^{\mathbb{R}}$ is the Euclidean norm. Taking $\Lambda\left(f_{d}, \Gamma\right)=f\{R \mid \Xi, \Gamma\}$ as the likelihood function of $f_{d}$ and $\Gamma$ [8], their joint ML estimates can be obtained through

$$
\left(f_{d}^{\#}, \Gamma^{\#}\right)=\underset{\left(\hat{f}_{d}, \hat{\Gamma}\right)}{\arg \max }\left\{\Lambda\left(\hat{f}_{d}, \hat{\Gamma}\right)\right\},
$$

where $f_{d}^{\#}$ and $\Gamma^{\#}$ stand for the ML estimates of $f_{d}$ and $\Gamma$, while $\hat{f}_{d}$ and $\hat{\Gamma}$ denote the trial values of $f_{d}$ and $\Gamma$, respectively. Since $f_{d}$ and $\Gamma$ are decoupled, this is a separable estimation problem which can be solved in two steps [9-10].
Firstly, $\hat{f}_{d}$ is regarded as a constant and it is found that (4) reaches its maximum when

$$
\hat{\Gamma}=\Xi^{H} R \text {. }
$$

Secondly, (6) is substituted into $\Lambda\left(\hat{f}_{d}, \hat{\Gamma}\right)$ and the likelihood function becomes

$$
\left.\Lambda\left(\hat{f}_{d}, \hat{\Gamma}\right)\right|_{\hat{\Gamma}=\Xi^{H} R}=\left(\pi \sigma^{2}\right)^{-N} \exp \left\{\frac{\left(2 N^{-1}-N^{-2}\right) R^{H} \Xi \Xi^{H} R-R^{H} R}{\sigma^{2}}\right\} .
$$

As the right-hand side of (7) is independent of $\hat{\Gamma}$, it coincides with the marginal likelihood function of $\hat{f}_{d}$. Obviously, maximizing (7) is equivalent to maximizing the inner term of $R^{H} \Xi \Xi^{H} R$. Thus, the ML estimate for $f_{d}$ can be expressed as

$$
f_{d}^{\#}=\arg \max _{\hat{f}_{d}}\left\{R^{H} P_{\Xi} R\right\} .
$$

where $P_{\Xi}=\Xi \Xi^{H}$. Due to the very form of $e_{i}$, different columns of $\Xi$ are mutually orthogonal. Hence, $P_{\Xi}$ can be decomposed into

$$
P_{\Xi}=\sum_{i=0}^{M-1} e_{i} e_{i}^{H} .
$$

Additionally, we define the periodogram of signal $R$ as

$$
\rho(f)=\left|\sum_{m=0}^{N-1} r_{m} \exp (-j 2 \pi m f / N)\right|^{2} .
$$

Substituting (9) into (8) yields

$$
f_{d}^{\#}=\arg \max _{\hat{f}_{d}}\left\{\sum_{i=0}^{M-1} \rho\left(f_{d, i}\right)\right\},
$$

where $f_{d, i} \stackrel{\Delta}{=} i+\hat{f}_{d}$. The relationship between the periodogram of the received signal and the maximum likelihood estimate of $f_{d}$ is clearly established. Since $\rho(f) \equiv \rho(f+N)$, in the sequel we shall consider the scenario where $f_{d} \in(-N / 2, N / 2]$ only and introduce a practical realization for (11).

\section{REALIZATION OF ML ESTIMATOR}

\section{A. Sub-optimal ML Estimator}

Although (11) gives the close-form expression for the ML estimate of $f_{d}$, its evaluation is not straightforward. Nevertheless, it is worth noting from (10) that (11) can be implemented through FFT when $\hat{f}_{d} \in \mathrm{Z}$, where $\mathrm{Z}$ denotes the set of integers. Moreover, the technique of interpolation can be employed to approximate $\rho\left(f_{d, i}\right)$ when $\hat{f}_{d} \notin \mathrm{Z}$. Considering this, a sub-optimal MLE is presented in this section. First of all, we construct a zero-padded sequence $\left\{r_{m}^{\prime}\right\}$ :

$$
r_{m}^{\prime}=\left(\begin{array}{ll}
r_{m}, & 0 \leq m \leq N-1 ; \\
0, & N \leq m \leq L N-1 ;
\end{array}\right.
$$

where the oversize ratio $L=2^{l^{\prime}}$ and the design parameter $l^{\prime}$ is a positive integer. Next, we invoke $L N$-point FFT over $\left\{r_{m}^{\prime}\right\}$, yielding 


$$
\Re(k)=\sum_{m=0}^{L N-1} r_{m}^{\prime} \exp [-j 2 \pi m k /(L N)],-L N / 2<k \leq L N / 2 .
$$

The sub-optimal MLE of $f_{d}$ then takes the form of

$$
\tilde{f}_{d}^{\#}=L^{-1} \underset{\hat{k}}{\arg \max }\left\{|\Re(k)|^{2} \sum_{i=0}^{M-1} \delta(k-\hat{k}-i L)\right\} \text {. }
$$

Using (2) and ignoring the term $\xi, \Re(k)$ can be rewritten as

$$
\Re(k)=\sum_{l=0}^{M-1} h_{l} p_{l} \exp \left[j \omega_{l}(N-1)\right] \sin \left(N \omega_{l}\right) / \sin \left(\omega_{l}\right),
$$

where $\omega_{l}=\pi(L N)^{-1}\left[L\left(f_{d}+l\right)-k\right]$. When $f_{d}=\lambda L$ and $\lambda \in Z$, it turns out that

$$
\underset{\hat{f}_{d}}{\arg \max }\left\{\sum_{i=0}^{M-1} \rho\left(f_{d, i}\right)\right\}=L^{-1} \underset{\hat{k}}{\arg \max }\left\{\sum_{i=0}^{M-1}|\Re(\hat{k}+i L)|^{2}\right\} .
$$

In this case, the sub-optimal estimator (14) agrees with the optimal estimator (11), i.e. $\tilde{f}_{d}^{\#}=f_{d}^{\#}$. When $f_{d} \neq \lambda L$, (16) no longer holds but (14) is still able to give the best approximation to (11) from the set of $\{-N / 2+1 / L,-N / 2+2 / L, \ldots$ $N / 2\}$. As a result, we have $\left|\tilde{f}_{d}^{\#}-f_{d}\right| \leq(2 L)^{-1}$. Evidently, the estimation accuracy is proportional to $L$. On the other hand, increasing $L$ also means increasing the size of FFT, which is undesirable for practical implementation. Thus, there is a trade off between the desired precision and the computation simplicity, and (14) can be utilized to provide a coarse estimate of $f_{d}$ when $L$ is limited.

\section{B. Preamble Design and Simplified Sub-optimal Estimator}

Considering the sensitivity of the OFDM system to frequency error, we introduce a fine estimation scheme to recover the part of CFO that cannot be accomplished by (14). In the foregoing discussions, symbol $P$ is presumed to be known by the receiver. Practically, it can be regarded as the preamble. For simplicity, we assume $P$ is a binary sequence with $p_{m} \in\{0,1\}$. Under such circumstances, $P$ corresponds to the assignment of the pilot tones. For the objective of achieving a large estimation range, the ambiguity about $f_{d}$ should be avoided and the maxima of the likelihood function should be unique within the desired range. Moreover, to suppress the ACI caused by neighboring tones, the distance between two adjacent pilot tones should be sufficiently wide.

Taking both requirements into account, we propose to employ a binary PN code $C=\left\{\begin{array}{lllll}c_{1} & c_{2} & \ldots & c_{K}\end{array}\right\}$ in arranging $P$, which satisfies the condition of $K+K^{\prime} Q_{l}=M$, where $K^{\prime}$ is the size of the subset $\left\{c_{m}^{\prime} \mid c_{n}^{\prime} \in C\right.$ and $\left.c_{m}^{\prime}=1\right\}$, and $Q_{l}>1$ is a positive integer. The desired $P$ is then obtained by inserting $Q$ consecutive zeros after each $c_{m}^{\prime}$. Supposing $f_{d}=\lambda L+v$, where $|v| \leq(2 L)^{-1}$, for $\lambda+L\left(m_{i}-1\right)<k<\lambda+L\left(m_{i}+1\right)$, the module of (15) can be approximated by $|\mathfrak{R}(k)| \approx\left|h_{m_{i}}\right| \sin \left(N \omega_{i}\right) / \sin \left(\omega_{i}\right)$, where $\omega_{i}=\pi\left[L\left(f_{d}+m_{i}\right)-k\right](L N)^{-1}$. Particularly, for $k=\lambda+L\left(m_{i} 1 / 2\right)$ and $\left.\lambda+L(m+1 / 2), \quad\left|\Re\left[\lambda+L\left(m_{i}-1 / 2\right)\right]\right| \approx-\cos \left(\frac{\pi v}{L}\right)\left|h_{m_{i}}\right| \frac{\pi v}{N L}-\frac{\pi}{2 N}\right)^{-1} \quad$ and $\left|\Re\left[\lambda+L\left(m_{i}+1 / 2\right)\right]\right| \approx \cos \left(\frac{\pi v}{L}\right)\left|h_{m_{i}}\right|\left(\frac{\pi v}{N L}+\frac{\pi}{2 N}\right)^{-1}$. After some simple arithmetic manipulations, $v$ can be approximated by
$\frac{1-\left.\left|\Re\left[\lambda+L\left(m_{i}+1 / 2\right)\right]\right| \cdot \Re\left[\lambda+L\left(m_{i}-1 / 2\right)\right]\right|^{-1}}{2\left(1+\left|\Re\left[\lambda+L\left(m_{i}+1 / 2\right)\right]\right| \cdot \mid \Re\left[\lambda+\left.L\left(m_{i}-1 / 2\right)\right|^{-1}\right)\right.}$. Considering the presence of noise, a more robust estimator for $v$ takes the form of

$$
\tilde{v}=\frac{1}{K^{\prime}} \sum_{i=1}^{K^{\prime}} \frac{1-\left|\Re\left[\lambda+L\left(m_{i}+1 / 2\right)\right]\right| \cdot\left|\Re\left[\lambda+L\left(m_{i}-1 / 2\right)\right]\right|^{-1}}{2\left(1+\left|\Re\left[\lambda+L\left(m_{i}+1 / 2\right)\right]\right|\left|\Re\left[\lambda+L\left(m_{i}-1 / 2\right)\right]\right|^{-1}\right.} .
$$

Besides, as a result of the proposed $P$, we have

$$
\begin{aligned}
\underset{\hat{k}}{\arg \max }\left\{\sum_{i=0}^{M-1}\left|\Re\left(\hat{k}+i^{\prime} L\right)\right|^{2}\right\} & \approx \underset{\hat{k}}{\arg \max }\left\{\left|\frac{\sin \left(N \sigma_{\hat{k}}\right)}{\sin \left(\sigma_{\hat{k}}\right)}\right|^{2} \sum_{i=1}^{K^{\prime}}\left|h_{m_{i}}\right|^{2}\right\} \\
& \approx \underset{\hat{k}}{\arg \max }\left\{\left|\frac{\sin \left(N \sigma_{\hat{k}}\right)}{\sin \left(\sigma_{\hat{k}}\right)}\right| \sum_{i=1}^{K^{\prime}}\left|h_{m_{i}}\right|\right\} .
\end{aligned}
$$

where $\varpi_{\hat{k}}=\pi(L N)^{-1}\left(L f_{d}-\hat{k}\right)$. Therefore, the square operator involved in calculating the periodogram can be dropped, and (14) is simplified into

$$
\tilde{f}_{d}^{\#}=L^{-1} \arg \max _{\hat{k}}\left\{|\Re(k)| \sum_{i=1}^{K^{\prime}} p_{m_{i}} \delta\left(k-\hat{k}-m_{i} L\right)\right\} .
$$

The CFO estimator presented by [7] agrees with (19), which is a special case of the general formula (14) under the above assumptions about $P$. Eventually, the estimation of $f_{d}$ can be obtained by combining (17) and (19):

$$
\begin{aligned}
& \tilde{f}_{d}=\tilde{f}_{d}^{\#}+\tilde{v} . \\
& \text { IV. PERFORMANCE ANALYSIS }
\end{aligned}
$$

\section{A. Estimation Range of the Proposed Algorithm}

It is evident from (11) that the maximum range within which no ambiguity occurs is limited to a period of length $N$. Because of the auto-correlation properties of the PN code that is used in arranging $P$, the solution of (19) is unique as far as the range $(-N / 2, N / 2]$ is concerned. Therefore, the estimation range of algorithm (20) equals $(-N / 2, N / 2]$.

\section{B. Statistical Properties of the ML Estimator}

For the simplicity of analysis, here we only investigate the statistical properties of the MLE given by (11). Letting $\mu\left(\hat{f}_{d}\right)=\left.\left\|P_{\Xi} R\right\|^{2}\right|_{f_{d}=\hat{f}_{d}}$ and assuming SNR $\gg 1$, the expectation and variance of $f_{d}^{\#}$ can be approximated by

$$
E\left\{f_{d}^{\#}\right\} \approx f_{d}-\left.E\left\{\mu^{\prime}\left(\hat{f}_{d}\right)\right\}\left[E\left\{\mu^{\prime \prime}\left(\hat{f}_{d}\right)\right\}\right]^{-1}\right|_{\hat{f}_{d}=f_{d}}
$$

and

$$
\left.\operatorname{var}\left\{f_{d}^{\#}\right\} \approx E\left\{\left[\mu^{\prime}\left(\hat{f}_{d}\right)\right]^{2}\right\}\left[E\left\{\mu^{\prime \prime}\left(\hat{f}_{d}\right)\right\}\right]^{-2}\right|_{\hat{f}_{d}=f_{d}},
$$

respectively, where $\mu^{\prime}\left(\hat{f}_{d}\right)=\frac{\partial\left[\mu\left(\hat{f}_{d}\right)\right]}{\partial\left(\hat{f}_{d}\right)}$ and $\mu^{\prime \prime}\left(\hat{f}_{d}\right)=\frac{\partial^{2}\left[\mu\left(\hat{f}_{d}\right)\right]}{\partial^{2}\left(\hat{f}_{d}\right)}$. Noting $W_{M}^{H} W_{M}=N I_{M}$ and $E\left\{\Omega\left(-f_{d}\right) \xi \xi^{H} \Omega\left(f_{d}\right)\right\}=\sigma^{2} I_{N}$, we obtain

$$
E\left\{\left[\mu^{\prime}\left(f_{d}\right)\right]^{2}\right\}=8 \pi^{2} N^{-2} \sigma^{2} \Gamma^{H} W_{M}^{H} T\left(N I_{N}-P_{W}\right) T W_{M} \Gamma
$$
and

$$
E\left\{\mu^{\prime \prime}\left(f_{d}\right)\right\}=8 \pi^{2} N^{-2} \Gamma^{H} W_{M}^{H} T\left(N I_{N}-P_{W}\right) T W_{M} \Gamma .
$$


where $T=\operatorname{diag}\{0,1, \ldots N-1\}$ and $P_{W}=\sum_{i=0}^{M-1} w_{i} w_{i}^{H}$. Exploiting

(23) and (24), (21) and (22) can finally be reduced to

$$
\begin{gathered}
E\left\{f_{d}^{\#}\right\} \approx f_{d}, \\
\operatorname{var}\left\{f_{d}^{\#}\right\} \approx 4 \pi^{2} N^{-2}\left\{E\left[\bar{\xi}^{H} \Theta \bar{\zeta}\right]+2 \sigma^{2} \Gamma^{H} W_{M}^{H} \Theta W_{M} \Gamma\right\},
\end{gathered}
$$

where $\Theta=2 T P_{W} T-P_{W} T^{2}-\mathrm{T}^{2}$ and $\bar{\zeta}=\Omega\left(-f_{d}\right) \zeta$. When SNR is sufficiently high, the first term in the bracket of (26) is negligible, yielding

$$
\operatorname{var}\left\{f_{d}^{\#}\right\} \approx 8 \pi^{2} N^{-2} \sigma^{2} \Gamma^{H} W_{M}^{H} \Theta W_{M} \Gamma
$$

In [9], the general formula of computing the Fisher information matrix is given, which can be used to derive the $\mathrm{CRB}$ for the estimation of a vector parameter. As the CRB places a lower bound on the variance of each element to be estimated, it can be applied as a benchmark in evaluating the performance of the unbiased estimators. For this reason, we define the vector parameter of (6) as $\alpha=\left[\operatorname{Re}\left(H_{f}\right), \operatorname{Im}\left(H_{f}\right), f_{d}\right]^{T}$, where $\operatorname{Re}($.$) and \operatorname{Im}($.$) denote the real and imaginary parts of$ the enclosed quantity, respectively. Then the Fisher information matrix $F$ can calculated via

$$
[F]_{i_{1}, i_{2}}=E\left\{\frac{\partial^{2}\left[\ln \Lambda\left(f_{d}, H_{f}\right)\right]}{\partial \alpha_{i_{1}} \alpha_{i_{2}}}\right\}, 1 \leq i_{1}, i_{2} \leq 2 M+1
$$

where []$_{i_{1}, i_{2}}$ represents the element on the $i_{1}$ th row and the $i_{2}$ th column of the inner matrix. The CRB of $f_{d}$ should be

$$
C R B\left(f_{d}\right)=\left[F^{-1}\right]_{2 M+1,2 M+1} \text {. }
$$

Employing the approach in Appendix B of [8], (29) is found to be

$$
C R B\left(f_{d}\right)=8 \pi^{2} N^{-2} \sigma^{2} \Gamma^{H} W_{M}^{H} \Theta W_{M} \Gamma .
$$

Obviously, (30) coincides with (27), indicating the variance of the proposed MLE approximates the CRB and is asymptotically efficient.

\section{Simulation Results}

Following assumptions have been made in the conducted computer simulations: the pulse shaping is performed through a raised cosine roll-off filter and the roll-off factor is 0.5; symbol timing synchronization have been accomplished already; the channel is modeled as a typical urban channel with six paths, and the channel response is the same as that appearing in [8]; the PN code used for pilot tone allocation is generated from the m-sequence; the SNR is defined by $\|\Gamma\|^{2} \sigma^{-2}$.

Assuming SNR $=10 \mathrm{~dB}$, Table. 1 gives the coarse estimation results of (19) for $f_{d}=\{0.63,-9.5,20.44,-58.185,100.773,-120.099,-255$, $256\}$ and $L=\{2,4,8,16\}$. The other parameters used in this simulation are: $N=512, M=500, Q_{l}=3, K=200$ and $K^{\prime}=100$. It

\begin{tabular}{|c|c|c|c|c|c|c|c|c|}
\hline$f_{d}$ & 0.63 & -9.5 & 20.44 & -58.185 & 100.773 & -120.099 & -255 & 256 \\
\hline 2 & 0.5 & -9.5 & 20.5 & -58 & 101 & -120 & -255 & 256 \\
\hline 4 & 0.75 & -9.5 & 20.5 & -58.25 & 100.75 & -120 & -255 & 256 \\
\hline 8 & 0.625 & -9.5 & 20.5 & -58.125 & 100.75 & -120.125 & -255 & 256 \\
\hline 16 & 0.625 & -9.5 & 20.4375 & -58.1875 & 100.75 & -120.125 & -255 & 256 \\
\hline
\end{tabular}
can be obtained from this table that the estimation range is ($N / 2, N / 2]$. Besides, for each pair of $\left\{L, f_{d}\right\}$ being considered, the relationship of $\left|\tilde{f}_{d}^{\#}-f_{d}\right| \leq(2 L)^{-1}$ always holds. Therefore,
TABLE 1

CoArse Estimation Results For DifFERENT $L$ AND $f_{d}$

the validity of the simplified coarse estimator given by (19) is demonstrated.

Once the coarse estimation $\tilde{f}_{d}^{\#}$ has been obtained, the remaining part of $f_{d}-\tilde{f}_{d}^{\#}$ can be estimated by the use of (17). To measure the accuracy of the integral estimation given by (20), we introduce two new variables: the average bias $\eta$ and the mean square error $\gamma$, which are defined by $\eta=f_{d}-\left(N_{S}\right)^{-1} \sum_{i=1}^{N_{S}}\left(\tilde{f}_{d}\right)_{i}$ and $\gamma=\left(N_{S}\right)^{-1} \sum_{i=1}^{N_{S}}\left[\left(\tilde{f}_{d}\right)_{i}-f_{d}\right]^{2}$, where $N_{S}$ denotes the number of simulations and $\left(\tilde{f}_{d}\right)_{i}$ represent the outcome of the $i$ th run. As functions of SNR, $\eta$ and $\gamma$ are illustrated in Fig.2 and Fig.3, respectively, with $L$ as a parameter. The CFO is set to $f_{d}=-58.185$ and $N_{s}=10^{3}$. The other system parameters used in these simulations are the same as those used in obtaining Table.1. It is clear from these two figures that the accuracy of the proposed algorithm is proportional to the oversize ratio $L$. When $L$ is not large enough, notwithstanding the high level of SNR, it is noted from Fig. 2 that the CFO estimation is biased and the error floor also occurs in the curve of mean square error in Fig.3. This is due to the quantization effects of FFT, which can be overcome by raising $L$. When $L$ is sufficiently large, it is found that the estimator (20) is asymptotically unbiased and its variance approaches the CRB closely.

\section{CONCLUSION}

In this paper, a MLE for the CFO has been presented for OFDM applications. The derived likelihood function has revealed the relationship between the CFO and the periodogram of the received signal. Theoretical analysis has shown that the presented MLE is unbiased and its variance approximates the CRB. To realize this MLE in practice, a sub-optimal estimator has been developed in which zeropadded FFT is invoked for implementation. To broaden the estimation range as well as to reduce the implementation complexity, a preamble structure comprising nonuniformlyspaced pilot tones has been introduced and PN code has been employed for the assignment of pilot tones. Based on this preamble, the estimation process has been split into two phases: the coarse estimate has been obtained by the 
correlation between the received spectrum and the original pattern of the preamble; while the fine estimate has been obtained by investigating the magnitude attenuation of the frequency bins around those CFO-shifted pilot tones. Both theoretical analysis and computer simulations has indicated that the accuracy of this simplified estimator is proportional to the oversize ratio of zero-padded FFT and its estimation range equals the bandwidth of OFDM signal. When the oversize ratio is sufficiently high, the performance of the proposed sub-optimal estimator approaches that of the proposed MLE.

\section{REFERENCES}

[1] T. Pollet, M. Blade and M. Moeneclaey, "BER sensitivity of OFDM systems to carrier frequency offset and Weiner phase noise," IEEE Trans. Commun., vol. 43, pp. 191-193, Feb./Mar./Apr. 1995.

[2] T. M. Schmidl and D. C. Cox, "Robust frequency and timing synchronization for OFDM," IEEE Trans. Commun., vol. 45, pp. 16131621, Dec. 1997.

[3] M. Morelli, A. N. Andrea and U. Mengali, "Frequency resolution in OFDM systems," IEEE Commun. Letters, vol. 4, pp. 134-136, Apr. 2000 .

[4] M. Luise and R. Reggiannini, "Carrier frequency acquistion and tracking for OFDM systems," IEEE Trans. Commun. , vol. 44, pp. 15901598, Nov. 1996.

[5] M. Morelli and U. Mengali, "An improved frequency offset estimator for OFDM applications," IEEE Commun. Letters, vol. 3, pp. 75-77, Mar. 1999.

[6] H. K. Song, Y. H. You, J. H. Paik and Y. S. Cho, "Frequency-offset synchronization and channel estimation for OFDM-based transmissions," IEEE Commun. Letters, vol. 4, pp. 95-97, Mar. 2000.

[7] H. Nogami and T. Nagashima, "A frequency acquisition technique for OFDM systems," Proceeding of PIMRC'95, pp. 1010-1015, Sept. 1995.

[8] M. Morelli and U. Mengali, "Carrier-frequency estimation for transmissions over selective channels," IEEE Trans. Commun. , vol. 48, pp. 1580-1589, Sept. 2000.

[9] S. M. Kay, Fundamentals of Statistical Signal Processing: Estimation Theory. Englewood Cliffs, NJ: Prentice-Hall, 1993.
[10] G. H. Golub and V. Pereyra, "The differentiation of pesudo-inverses and nonlinear least square problems whose variables separate," Journal of the society for industrial and applied mathematics, series B: numerical analysis, pp. 413-432, vol.10, No.2, Apr. 1973.

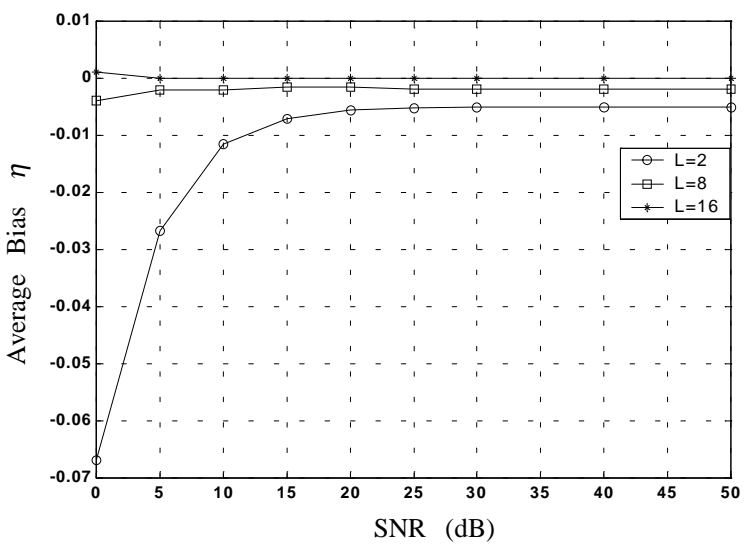

Fig.2 Average bias of the proposed algorithm for different oversize ratio

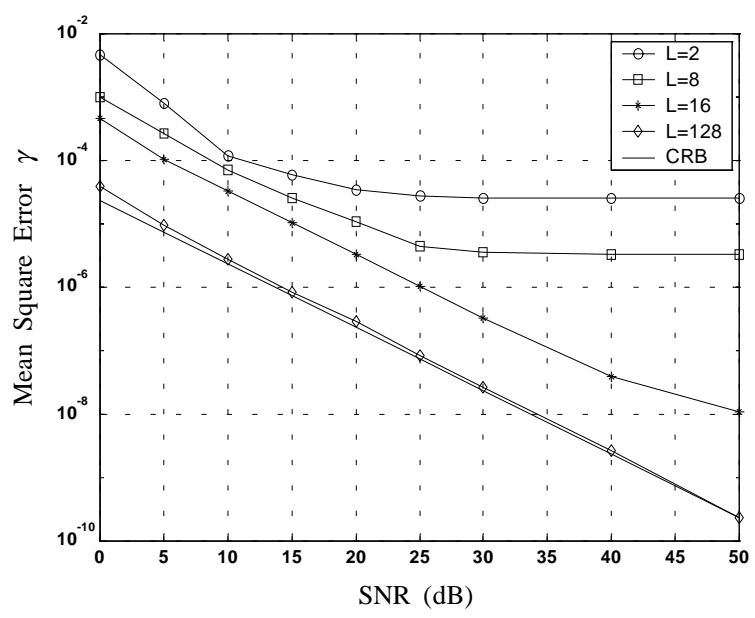

Fig.3 Mean square error of the proposed algorithm for different oversize ratio 